\title{
The Boolean Model in the Shannon Regime: Three Thresholds and Related Asymptotics
}

\author{
Venkat Anantharam \\ EECS Department \\ University of California, Berkeley \\ ananth@eecs.berkeley.edu \\ François Baccelli \\ Department of Mathematics and ECE Department \\ University of Texas, Austin \\ and \\ INRIA/ENS, Paris, France \\ francois.baccelli@austin.utexas.edu or francois.baccelli@ens.fr \\ July 28, 2018
}

\begin{abstract}
Consider a family of Boolean models, indexed by integers $n \geq 1$, where the $n$-th model features a Poisson point process in $\mathbb{R}^{n}$ of intensity $e^{n \rho_{n}}$ with $\rho_{n} \rightarrow \rho$ as $n \rightarrow \infty$, and balls of independent and identically distributed radii distributed like $\bar{X}_{n} \sqrt{n}$, with $\bar{X}_{n}$ satisfying a large deviations principle. It is shown that there exist three deterministic thresholds: $\tau_{d}$ the degree threshold; $\tau_{p}$ the percolation threshold; and $\tau_{v}$ the volume fraction threshold; such that asymptotically as $n$ tends to infinity, in a sense made precise in the paper: (i) for $\rho<\tau_{d}$, almost every point is isolated, namely its ball intersects no other ball; (ii) for $\tau_{d}<\rho<\tau_{p}$, almost every ball intersects an infinite number of balls and nevertheless there is no percolation; (iii) for $\tau_{p}<\rho<\tau_{v}$, the volume fraction is 0 and nevertheless percolation occurs; (iv) for $\tau_{d}<\rho<\tau_{v}$, almost every ball intersects an infinite number of balls and nevertheless the volume fraction is 0 ; (v) for $\rho>\tau_{v}$, the whole space
\end{abstract}


covered. The analysis of this asymptotic regime is motivated by related problems in information theory, and may be of interest in other applications of stochastic geometry.

\section{Introduction}

The Boolean model was considered in high dimensions in a few papers, both within the framework of stochastic geometry [5, 8] and within the framework of information theory [1]. The present paper discusses three thresholds and some asymptotics related to these thresholds in a setting analogous to that in [1], which is that where the radii of the balls in the Boolean model scale with the dimension $n$ of the ambient space like $\bar{X}_{n} \sqrt{n}$, where $\left(\bar{X}_{n}, n \geq 1\right)$ is a sequence of random variables. In this paper, we assume that this sequence of random variables satisfies a large deviations principle (LDP).

The first threshold is the volume fraction threshold, which is the threshold at which the probability of coverage of the origin by the Boolean model switches from asymptotically vanishing to asymptotically approaching 1 as the dimension $n$ tends to $\infty$. The second one is the percolation threshold; it was first studied in detail in [8] in the particular case where $\bar{X}_{n}$ is a constant. The case with random $\bar{X}_{n}$ was also discussed in [5]. This is the threshold at which the probability of percolation in the Boolean model switches from asymptotically approaching 0 to being asymptotically nonzero as $n \rightarrow \infty$. The last is the degree threshold. This is the threshold at which the mean number of grains of the Palm version of the Boolean model that intersect the grain of the origin switches from asymptotically being finite to asymptotically approaching $\infty$ as $n \rightarrow \infty$. It is not hard to see that these three thresholds are decreasing in the order in which they were presented.

The main new contributions of the present paper are (a) representations of these three thresholds in terms of optimization problems based on the rate function of the LDP and (b) explicit asymptotics for various rates of convergence in the neighborhood of these thresholds.

\section{Setup}

In each dimension $n \geq 1$ we have a homogeneous Poisson process of intensity $e^{n \rho_{n}}$ (i.e. normalized logarithmic intensity $\rho_{n}$ ). Assume that $\rho_{n} \rightarrow \rho$ as $n \rightarrow \infty$. Note that $\rho$ is a real number (which can be negative). It is called the asymptotic 
normalized logarithmic intensity of this sequence of Poisson processes. We will assume that the processes are defined on a single probability space $(\Omega, \mathcal{F}, \mathbb{P})$, although the coupling between the different dimensions is not relevant for the issues we consider. We will denote by $\mathbb{P}_{n}^{0}$ the Palm probability of the Poisson point process in dimension $n$. See [3, Chapter 13] for the definition of and basic facts about Palm probabilities.

To each point $T_{n}^{(k)}, k \geq 1$, of the Poisson process in dimension $n$ (enumerated in some way) we associate a mark $X_{n}^{(k)} \in \mathbb{R}^{+}$. The $X_{n}^{(k)}, k \geq 1$, are assumed to be independent and identically distributed (i.i.d.) and independent of the points. For each dimension $n$, let $X_{n}^{(k)} \stackrel{d}{=} \bar{X}_{n}$ for all $k$. Let $R_{n}^{*}$ denote $\mathbb{E}\left[\bar{X}_{n}\right]$, and let $R^{*}:=\lim _{n \rightarrow \infty} R_{n}^{*}$, where the last limit is assumed to exist. We assume that $0<R^{*}<\infty$. We assume that the sequence $\left(\bar{X}_{n}, n \geq 1\right)$ satisfies an LDP, with good and convex rate function $I(\cdot)$ [2], e.g. by assuming the Gärtner-Ellis conditions. We also assume that the following condition holds:

$$
\limsup _{n \rightarrow \infty} \mathbb{E}\left[\left(\bar{X}_{n}\right)^{\gamma n}\right]^{\frac{1}{n}}<\infty \quad \text { for some } \gamma>1
$$

By the deterministic setting we mean that $\bar{X}_{n}$ is deterministic and equal to $R_{n}^{*}$ for each $n \geq 1$, with $R_{n}^{*} \rightarrow R^{*}$ as $n \rightarrow \infty$. The deterministic setting is a special case of the general setting, but we will separately highlight the results in this case, since it is of particular interest.

To the marked point process in dimension $n$, we associate a Boolean model where the grain of point $T_{n}^{(k)}$ is a closed ball of radius $X_{n}^{(k)} \sqrt{n}$. Let

$$
\mathcal{C}_{n}:=\bigcup_{k} B\left(T_{n}^{(k)}, X_{n}^{(k)} \sqrt{n}\right)
$$

denote this Boolean model, with $B(t, r)$ denoting the closed ball of center $t \in \mathbb{R}^{n}$ and radius $r \geq 0$. Here, and in the rest of the paper, $:=$ denotes equality by definition.

From Slivnyak's theorem [3, Chapter 13], the Palm version of the process in each dimension $n$ (i.e. its law under $\mathbb{P}_{n}^{0}$ ) is equivalent in law to the superposition of a stationary version of the process and a process with a single point at the origin carrying a ball with radius having law $\bar{X}_{n} \sqrt{n}$, and independent of the stationary version (which is called the reduced process of the Palm version).

Our motivations for the analysis of this setting came from related problems in information theory that we studied recently [1]. More specifically, in the study of error probabilities for coding over an additive white Gaussian noise channel [4, 
Section 7.4], it is natural to consider a sequence of Poisson processes, one in each dimension $n \geq 1$, with well defined asymptotic logarithmic intensity, as was done in [1], motivated by the ideas in [9]. The error exponent questions studied in [1] are related to 1 the consideration of a Boolean model where the grains associated with the individual points are defined in terms of additive white Gaussian noise: for all $n \geq 1$ and $k \geq 1$, let $W_{n}^{(i, k)}, n \geq i \geq 1$, denote an i.i.d. sequence of Gaussian random variables, all centered and of variance $\sigma^{2}>0$. Let $W_{n}^{(k)}$ denote the $n$-dimensional vector with coordinates $W_{n}^{(i, k)}, n \geq i \geq 1$. Then $T_{n}^{(k)}+W_{n}^{(k)}$ belongs to the closed ball of center $T_{n}^{(k)}$ and radius $X_{n}^{(k)} \sqrt{n}$, with

$$
X_{n}^{(k)}:=\left(\frac{1}{n} \sum_{i=1}^{n}\left(W_{n}^{(i, k)}\right)^{2}\right)^{\frac{1}{2}}
$$

satisfying an LDP and all the assumptions listed above. For the error exponent problem what is of interest is not this Boolean model, but the related Boolean model where the grain associated to each point is not the random ball described above, but rather an associated typicality region, which in this case we may define as the set

$$
\left\{T_{n}^{(k)}+v: v \in \mathbb{R}^{n},\|v\|_{2} \leq \sigma \sqrt{n}+\alpha_{n}\right\},
$$

where $\|v\|_{2}$ denotes the usual Euclidean length of $v$ and $0<\alpha_{n}=O(\sqrt{n})$ are chosen such that 2

$$
\begin{aligned}
& \frac{\alpha_{n}}{\sqrt{n}} \rightarrow 0 \text { as } n \rightarrow \infty ; \\
& P\left(\left\|W_{n}^{(k)}\right\| \leq \sigma \sqrt{n}+\alpha_{n}\right) \rightarrow 1 \text { as } n \rightarrow \infty \text { (for each } 1 \leq k \leq n, \text { of course); } \\
& \frac{1}{n} \log \operatorname{Vol}\left\{v \in \mathbb{R}^{n}:\|v\|_{2} \leq \sigma \sqrt{n}+\alpha_{n}\right\} \rightarrow \frac{1}{2} \log \left(2 \pi e \sigma^{2}\right) \text { as } n \rightarrow \infty .
\end{aligned}
$$

This fits within the class of deterministic Boolean models considered in this paper. Thus, having carried out the analysis in [1], it was natural for us to become curious about the asymptotic properties in dimension of the sequence of Boolean

\footnotetext{
${ }^{1}$ The scenario considered in [1] goes beyond additive white Gaussian noise to consider a setting where the additive noise comes from sections of a stationary and ergodic process. The Boolean models that arise in the more general case involve grains, defined by the typicality sets of the noise process, that are not necessarily spherically symmetric. Even more generally, in [1] the underlying point process in each dimension is allowed to be an arbitrary stationary ergodic process (while still requiring the existence of an asymptotic logarithmic intensity).

${ }^{2}$ It is straightforward to check that it is possible to choose $\left(\alpha_{n}, n \geq 1\right)$ satisfying these requirements.
} 
models with the grains being balls whose radii obey a large deviations principle in the sense described above, and the current paper may be viewed as a start in that direction. In particular, it is to be hoped that this particular asymptotic regime, which is so natural from an information theoretic viewpoint, will also be of value in the applications of stochastic geometry in other domains of science and engineering.

The paper is structured as follows. We start with a summary of results and heuristic explanations in Section 3 . We then give proofs in Section 4 . For smoothness of exposition, we first discuss the volume fraction threshold, then the degree threshold, and finally the percolation threshold in each of these sections. Some concluding remarks, making connections between the issues addressed here and the information theoretic concerns of [1], are made in Section 5, where in particular the instantiation of our general results in the case of Gaussian grains is worked out in detail.

\section{Results}

\subsection{Volume Fraction Threshold}

Consider the stationary version of the marked Poisson process in each dimension. We are interested in the asymptotic behavior of the probability with which the origin is covered, namely $\mathbb{P}\left(\underline{0} \in \mathcal{C}_{n}\right)$. By stationarity, for any Borel set of $\mathbb{R}^{n}$, this probability is also the mean fraction of the volume of the Borel set which is covered by the Boolean model. We claim that there is a number $\tau_{v}$, called the volume fraction threshold, such that for $\rho<\tau_{v}$ this probability asymptotically approaches 0 as $n$ tends to infinity, while for $\rho>\tau_{v}$ it asymptotically approaches 1. The value of $\tau_{v}$ depends on the large deviations rate function $I(\cdot)$ associated to the sequence of distributions of the radii of the marks.

The idea of the proof is based on the fact that most of the volume of a ball is at the boundary. Hence for all $R>0$, the mean number of points at distance roughly $R \sqrt{n}$ from the origin grows like

$$
e^{n \rho_{n}} e^{\frac{n}{2} \log (2 \pi e)+o(n)} e^{n \log R} .
$$

Each such point covers the origin with probability $\mathbb{P}\left(\bar{X}_{n} \geq R\right)$. For $R<R^{*}$ this probability is asymptotically 1 . For $R>R^{*}$ this probability decays like

$e^{-n I(R)+o(n)}$, where $I(\cdot)$ denotes the rate function for the convergence $\bar{X}_{n} \stackrel{\mathbb{P}}{\rightarrow} R^{*}$. 
Let $\underline{0}$ denote the origin in $\mathbb{R}^{n}$. We should therefore have

$$
\lim _{n \rightarrow \infty} \frac{1}{n} \log \mathbb{P}\left(\underline{0} \in \mathcal{C}_{n}\right)=\rho+\frac{1}{2} \log (2 \pi e)+\sup _{R \geq R^{*}}(\log R-I(R)),
$$

as long as

$$
\rho+\frac{1}{2} \log (2 \pi e)+\sup _{R \geq R^{*}}(\log R-I(R))<0,
$$

where we used the fact that $I\left(R^{*}\right)=0$ which implies that $\log (R) \leq \log \left(R^{*}\right)-$ $I\left(R^{*}\right)$ for $R \leq R^{*}$.

Also

$$
\lim _{n \rightarrow \infty} \mathbb{P}\left(\underline{0} \in \mathcal{C}_{n}\right)=1,
$$

if

$$
\rho+\frac{1}{2} \log (2 \pi e)+\sup _{R \geq R^{*}}(\log R-I(R))>0 .
$$

This gives a heuristic explanation of the value of the threshold in the following theorem:

Theorem 1. Under the foregoing assumptions, the volume fraction threshold is equal to

$$
\tau_{v}=-\frac{1}{2} \log (2 \pi e)+\inf _{R \geq R^{*}}(I(R)-\log R) .
$$

More precisely, for $\rho<\tau_{v}$, as $n$ tends to infinity, the volume fraction in dimension $n$, namely $\mathbb{P}\left(\underline{0} \in \mathcal{C}_{n}\right)$, tends to 0 exponentially fast with

$$
\lim _{n \rightarrow \infty} \frac{1}{n} \log \left(\mathbb{P}\left(\underline{0} \in \mathcal{C}_{n}\right)\right)=\rho-\tau_{v}
$$

whereas for $\rho>\tau_{v}$, it tends to 1 with

$$
\lim _{n \rightarrow \infty} \frac{1}{n} \log \left(-\log \mathbb{P}\left(\underline{0} \notin \mathcal{C}_{n}\right)\right)=\rho-\tau_{v} .
$$

Note that

$$
\tau_{v} \leq-\frac{1}{2} \log (2 \pi e)-\log R^{*}
$$

In the case of deterministic radii, i.e. when $\bar{X}_{n}$ equals the deterministic value $R_{n}^{*}$ for each $n \geq 1$, with $R_{n}^{*} \rightarrow R^{*}$ as $n \rightarrow \infty$, we have equality in eqn. (6). The R.H.S. of eqn. (6) is identical to what is called the Poltyrev threshold in [1], where it in effect arose in the context of the Boolean models with Gaussian 
grains truncated to their typicality regions, as described at the end of Section 2. In Section 5 we will discuss in more depth this connection between the questions addressed in this paper and the information theoretic questions studied in [1]. This threshold can also be described as follows: the volume of the $n$-ball of random radius $\bar{X}_{n} \sqrt{n}$ scales like $e^{n V+o(n)}$ as $n$ tends to infinity, for some constant $V$; we have $\tau_{v}=-V$ or equivalently the critical density $e^{n \tau_{v}+o(n)}$ scales like the inverse of the volume of this $n$-ball.

\subsection{Degree Threshold}

We are interested in the number $D_{n}$ of points other than $\underline{0}$ whose ball intersects the ball of the point at the origin under $\mathbb{P}_{n}^{0}$.

We claim that there is a number $\tau_{d}$, that we will call the degree threshold, such that if $\rho<\tau_{d}$, then $\mathbb{E}_{n}^{0}\left[D_{n}\right]$ asymptotically goes to 0 when $n$ tends to infinity, while for $\rho>\tau_{d}$ it asymptotically goes to $\infty$.

We argue as follows: condition on the radius of the ball of the point at the origin, call it $s \sqrt{n}$. Every point that lands in the ball of radius $s \sqrt{n}$ will have its ball meeting the ball of the origin. The number of such points grows like

$$
e^{n \rho_{n}} e^{\frac{n}{2} \log (2 \pi e)+o(n)} e^{n \log s} .
$$

Next consider points at a distance roughly $R \sqrt{n}$ from the (point at the) origin, with $R>s$. The number of such points grows like

$$
e^{n \rho_{n}} e^{\frac{n}{2} \log (2 \pi e)+o(n)} e^{n \log R} .
$$

Each such point has its ball intersecting the ball of the point at the origin with probability asymptotically equal to 1 if $R-s<R^{*}$ and with probability decaying like $e^{-n I(R-s)+o(n)}$ if $R-s>R^{*}$. The number of points meeting the ball of the origin, conditioned on this ball having radius $s \sqrt{n}$, therefore grows like

$$
e^{n\left(\rho+\frac{1}{2} \log (2 \pi e)+\sup _{R \geq s+R^{*}}(\log R-I(R-s))\right)+o(n)} .
$$

The probability that the ball of the origin has radius roughly $s \sqrt{n}$ decays like $e^{-n I(s)+o(n)}$. Thus, the overall growth rate of the number of points whose ball 
meets the ball of the origin is

$$
\begin{aligned}
& \sup _{s>0}\left(-I(s)+\rho+\frac{1}{2} \log (2 \pi e)+\sup _{R \geq s+R^{*}}(\log R-I(R-s))\right) \\
& =\rho+\frac{1}{2} \log (2 \pi e)+\sup _{s>0} \sup _{R \geq s+R^{*}}(-I(s)+\log R-I(R-s)) \\
& =\rho+\frac{1}{2} \log (2 \pi e)+\sup _{R>R^{*}}\left(\log R+\sup _{0<s \leq R-R^{*}}(-I(s)-I(R-s))\right) \\
& \stackrel{(a)}{=} \rho+\frac{1}{2} \log (2 \pi e)+\max _{R^{*} \leq R<2 R^{*}}\left(\log R-I\left(R-R^{*}\right)\right), \\
& =\rho+\frac{1}{2} \log (2 \pi e)+\sup _{R \geq 2 R^{*}}\left(\log R-2 I\left(\frac{R}{2}\right)\right),
\end{aligned}
$$

where in step (a) we have used the convexity of the rate function $I(\cdot)$ and the fact that $I\left(R^{*}\right)=0$, and in the last step we have observed that the maximum in the first of the terms in the overall maximum occurs at $R=2 R^{*}$. This gives intuition for the value of the threshold in the following theorem:

Theorem 2. Under the conditions of Theorem $\square$ the degree threshold is

$$
\tau_{d}=-\frac{1}{2} \log (2 \pi e)+\inf _{R \geq 2 R^{*}}\left(2 I\left(\frac{R}{2}\right)-\log R\right) .
$$

That is, for $\rho<\tau_{d}$, as $n$ tends to infinity, in dimension $n, \mathbb{E}_{n}^{0}\left[D_{n}\right]$ tends to 0 exponentially fast, whereas for $\rho>\tau_{d}$ it tends to infinity exponentially fast. In both cases,

$$
\lim _{n \rightarrow \infty} \frac{1}{n} \log \left(\mathbb{E}_{n}^{0}\left[D_{n}\right]\right)=\rho-\tau_{d} .
$$

It is sometimes more convenient to write the degree threshold as

$$
\tau_{d}=-\frac{1}{2} \log (2 \pi e)+\inf _{R \geq R^{*}}(2 I(R)-\log (2 R)) .
$$

Note that

$$
\tau_{d} \leq-\frac{1}{2} \log (2 \pi e)-\log \left(2 R^{*}\right)
$$


and that the R.H.S. of the last inequality is the degree threshold in the case of deterministic radii [8].

In the general case, the degree threshold can be described as follows: let $\bar{X}_{n}^{\prime}$ be an independent random variable with the same law as $\bar{X}_{n}$. The volume of the $n$ ball of random radius $\left(\bar{X}_{n}+\bar{X}_{n}^{\prime}\right) \sqrt{n}$ scales like $e^{n V+o(n)}$ as $n$ tends to infinity for some constant $V$; we have $\tau_{d}=-V$ or equivalently the critical density $e^{n \tau_{d}+o(n)}$ scales like the inverse of the volume of this $n$-ball.

\subsection{Percolation Threshold}

Consider the Palm version of the process in dimension $n$. Consider the connected component of $\mathcal{C}_{n}$ that contains the origin, called the cluster of the origin, and denote the set of points of the underlying Poisson process that lie in this connected component by $K_{n}$. The percolation probability in dimension $n$ is denoted by

$$
\theta_{n}:=\mathbb{P}_{n}^{0}\left(\left|K_{n}\right|=\infty\right)
$$

with $|A|$ the cardinality of set $A$. This is one of the standard definitions for percolation probability in continuum percolation theory, see [7, Section 1.4].

We are interested in the asymptotics of the percolation probability as $n \rightarrow \infty$. We claim that there is a number $\tau_{p}$, called the percolation threshold, such that for $\rho<\tau_{p}$ we have $\theta_{n} \rightarrow 0$ as $n \rightarrow \infty$, while for $\rho>\tau_{p}$ we have $\liminf _{n} \theta_{n}>0$.

Proposition 3. In the case of deterministic radii, the percolation and the degree thresholds coincide, i.e. $\tau_{p}=\tau_{d}$.

To see that $\tau_{p} \geq \tau_{d}$, note that if $\rho<\tau_{d}$ then $\mathbb{E}_{n}^{0}\left[D_{n}\right] \rightarrow 0$ as $n \rightarrow \infty$ from Theorem 2, It follows that $\mathbb{P}_{n}^{0}\left(D_{n}=0\right) \rightarrow 1$ as $n \rightarrow \infty$. Hence $\mathbb{P}_{n}^{0}\left(\left|K_{n}\right|=\right.$ $1) \rightarrow 1$ as $n \rightarrow \infty$, from which it follows that $\theta_{n} \rightarrow 0$ as $n \rightarrow \infty$. This means $\rho<\tau_{p}$. This argument actually works in the general case, i.e. it does not require the assumption of deterministic radii.

To see that $\tau_{p} \leq \tau_{d}$ in the case of deterministic radii, we need to prove that if $\rho>\tau_{d}$ then $\lim \inf _{n} \theta_{n}>0$. To this end, let us recall the main result of [8]. In our notation, in [8] Penrose considers the sequence of Poisson Boolean models with deterministic radii $R_{n}^{*}=R^{*}$ for each $n \geq 1$, and with normalized logarithmic intensities $\rho_{n}^{y}$ defined via

$$
e^{n \rho_{n}^{y}} \frac{(\pi n)^{\frac{n}{2}}}{\Gamma\left(\frac{n}{2}+1\right)}\left(2 R^{*}\right)^{n}=y, \text { for all } n \geq 1
$$


where $y>0$ is a fixed real number. Let $\theta_{n}^{y}$ denote the percolation probability in dimension $n$ with these choices. The main result [ 8 , Theorem 1] is that $\lim _{n \rightarrow \infty} \theta_{n}^{y}$ exists and equals the survival probability of a branching process with offspring distribution Poisson with mean $y$, and started with a single individual. In particular, this means that if $y>1$, then $\liminf _{n \rightarrow \infty} \theta_{n}^{y}>0$.

In our scenario with deterministic radii, the degree threshold (see eqn. (11)) is

$$
\tau_{d}=-\frac{1}{2} \log (2 \pi e)-\log \left(2 R^{*}\right), \quad(\text { deterministic radii }) .
$$

It suffices to observe that if $\rho>\tau_{d}$, then

$$
\lim _{n \rightarrow \infty} e^{n \rho_{n}} \frac{(\pi n)^{\frac{n}{2}}}{\Gamma\left(\frac{n}{2}+1\right)}\left(2 R_{n}^{*}\right)^{n}=\infty .
$$

That $\lim \inf _{n \rightarrow \infty} \theta_{n}>0$ then follows from the result of [8] cited above.

The main result on the case with random radii is:

Theorem 4. The percolation threshold is given by the formula

$$
\tau_{p}=-\frac{1}{2} \log (2 \pi e)+\inf _{R \geq R^{*}}\left(I(R)-\log \left(R+R^{*}\right)\right) .
$$

That is, for $\rho<\tau_{p}, \theta_{n} \rightarrow 0$ when $n$ tends to infinity, whereas for $\rho>\tau_{p}$ we have $\liminf \theta_{n}>0$.

Note that

$$
\tau_{p} \leq-\frac{1}{2} \log (2 \pi e)-\log \left(2 R^{*}\right) .
$$

In the case of deterministic radii the minimum in the expression for the percolation threshold in eqn. (13) is achieved at $R=R^{*}$ and so we have

$$
\tau_{p}=-\frac{1}{2} \log (2 \pi e)-\log \left(2 R^{*}\right), \quad \text { (deterministic radii) } .
$$

This also equals the value of the degree threshold in the case of deterministic radii.

The volume of the $n$-ball of random radius $\left(\bar{X}_{n}+R^{*}\right) \sqrt{n}$ scales like $e^{n V+o(n)}$ as $n$ tends to infinity, for some constant $V$; we have $\tau_{p}=-V$ or equivalently the critical density $e^{n \tau_{p}+o(n)}$ scales like the inverse of the volume of this $n$-ball. The intuition for this result is that what matters for percolation is the mean number of balls that intersect a ball with typical radius (namely roughly $R^{*} \sqrt{n}$ ): if $\rho<$ $\tau_{p}$, then on an event whose probability tends to 1 as $n$ tends to infinity, namely 
the event that the ball of the point at $\underline{0}$ has a radius in the interval $\left(R^{*} \sqrt{n}-\right.$ $\left.\alpha_{n}, R^{*} \sqrt{n}+\alpha_{n}\right)$ for appropriate $0<\alpha_{n}=O(\sqrt{n})$, no other ball intersects the latter ball asymptotically (because $\rho<\tau_{p}$ ) and hence there is no percolation. Conversely, for $\rho>\tau_{p}$, when the ball of $\underline{0}$ is at typicality, i.e. its radius lies in an interval of the kind defined above, we can consider a thinned version of the reduced process where we only retain points whose balls have radii that are at least above a threshold slightly less than the value of $R$ achieving the infimum in the definition of $\tau_{p}$ (assume for the moment that this infimum is achieved), and we will still have that the mean number of balls intersecting the ball of the origin tends to infinity like $e^{n \delta}$ with some $\delta>0$. Since these balls themselves have radius at least as big as the typical ball of the unconditional distribution, this scenario propagates via a supercritical branching process, implying asymptotic percolation. 3

\subsection{Ordering of the Thresholds}

Theorem 5. Under the foregoing assumptions,

$$
\tau_{d} \leq \tau_{p} \leq \tau_{v}
$$

Remark The ordering relation of the last theorem is not limited to the Poisson case. The family of Boolean models considered here can naturally be extended to a family of particle processes [11], where the $n$-th particle process features a stationary and ergodic point process $\mu_{n}$ in $\mathbb{R}^{n}$ with normalized logarithmic intensity $\rho_{n}$ such that $\rho_{n} \rightarrow \rho$ as $n \rightarrow \infty$, and i.i.d. marks satisfying the same independence and LDP assumptions as above. This family of particle processes will be said to admit a volume fraction threshold $\tau_{v}$ if the associated $\mathcal{C}_{n}$, still defined by eqn. (2), is such that $\mathbb{P}\left(\underline{0} \in \mathcal{C}_{n}\right)$ asymptotically approaches 0 as $n$ tends to infinity for $\rho<\tau_{v}$, while for $\rho>\tau_{v}$ it asymptotically approaches 1. Similarly, it will be said to admit a degree threshold $\tau_{d}$ if the Palm expectation of $D_{n}$ tends to 0 as $n$ tends to infinity for $\rho<\tau_{d}$, while for $\rho>\tau_{d}$ it tends to $\infty$. The definition of the percolation threshold can also be extended verbatim. Assuming that these three thresholds exist, then they must satisfy eqn. (14). This follows from first principles. If the volume fraction asymptotically tends to 1 , then percolation must hold asymptotically; hence $\tau_{p} \leq \tau_{v}$. If the mean number of balls that intersect the ball of the origin tends to 0 , then percolation cannot hold asymptotically; hence

\footnotetext{
${ }^{3}$ For technical reasons, the formal proof looks slightly different from this sketch, but this is the basic intuition.
} 
$\tau_{d} \leq \tau_{p}$

Returning to the Poisson case, to better understand the thresholds, we first need to recall some facts from basic convex analysis [10]. Since it is a good convex rate function, $I(\cdot)$ is proper, as defined in [10, pg. 24]. Further, since it is lower semicontinuous, its epigraph is closed [10, Thm. 7.1], so $I(\cdot)$ is closed in the sense of [10, pg. 52]. Recall that the domain of $I(\cdot)$, defined as the set of $R$ for which $I(R)$ is finite, is an interval, which is nonempty because $I\left(R^{*}\right)=0$. Since $I(\cdot)$ is closed and proper, the right and left derivatives, $I_{+}^{\prime}(\cdot)$ and $I_{-}^{\prime}(\cdot)$ respectively, are well-defined as functions on $\mathbb{R}$ (both defined to be $+\infty$ to the right of the domain of $I(\cdot)$ and to be $-\infty$ to the left of the domain of $I(\cdot))$. These are nondecreasing functions, each of which is finite on the interior of the domain of $I(\cdot)$, and satisfy ([10, Thm. 24.1]):

$$
I_{+}^{\prime}\left(z_{1}\right) \leq I_{-}^{\prime}(x) \leq I_{+}^{\prime}(x) \leq I_{-}^{\prime}\left(z_{2}\right), \text { if } z_{1}<x<z_{2},
$$

and, for all $x \in \mathbb{R}$,

$$
\lim _{z \uparrow x} I_{-}^{\prime}(z)=\lim _{z \uparrow x} I_{+}^{\prime}(z)=I_{-}^{\prime}(x) \text { and } \lim _{z \downarrow x} I_{-}^{\prime}(z)=\lim _{z \downarrow x} I_{+}^{\prime}(z)=I_{+}^{\prime}(x) .
$$

We further note that $0 \in\left[I_{-}^{\prime}\left(R^{*}\right), I_{+}^{\prime}\left(R^{*}\right)\right]$, since $I(\cdot)$ is a nonnegative function with $I\left(R^{*}\right)=0$.

This means we can define the following radii:

- $R_{v} \geq R^{*}$ as a value of $R$ satisfying

$$
\frac{1}{R_{v}} \in\left[I_{-}^{\prime}\left(R_{v}\right), I_{+}^{\prime}\left(R_{v}\right)\right]
$$

Such $R_{v}$ achieves the infimum in eqn. (3) for the volume fraction threshold. Further, since $R \mapsto \frac{1}{R}$ is strictly decreasing and decreases to 0 as $R \rightarrow \infty$, it follows that $R_{v}$ is uniquely defined and finite.

- $R_{d} \geq R^{*}$, as a value of $R$ satisfying

$$
\frac{1}{2 R_{d}} \in\left[I_{-}^{\prime}\left(R_{d}\right), I_{+}^{\prime}\left(R_{d}\right)\right]
$$

Such $R_{d}$ achieves the minimum in eqn. (11) for the degree threshold. Further, since $R \mapsto \frac{1}{2 R}$ is strictly decreasing and decreases to 0 as $R \rightarrow \infty$, it follows that $R_{d}$ is uniquely defined and finite. 
- $R_{p} \geq R^{*}$, as a value of $R$ satisfying

$$
\frac{1}{R_{p}+R^{*}} \in\left[I_{-}^{\prime}\left(R_{p}\right), I_{+}^{\prime}\left(R_{p}\right)\right] .
$$

Such $R_{p}$ achieves the minimum in eqn. (13) for the percolation threshold. Further, since $R \mapsto \frac{1}{R+R^{*}}$ is strictly decreasing and decreases to 0 as $R \rightarrow$ $\infty$, it follows that $R_{p}$ is uniquely defined and finite.

Theorem 6. With the foregoing definitions, we have

$$
R^{*} \leq R_{d} \leq R_{p} \leq R_{v} \leq R_{p}+R^{*} \leq 2 R_{d} .
$$

\section{Proofs}

\subsection{Proof of Theorem 1}

Below we will use the directed random geometric graph built as follows: its vertices are the nodes of the point process and there is an edge from $T_{n}^{(k)}$ to $T_{n}^{(l)}$, $l \neq k$ if $T_{n}^{(l)} \in B\left(T_{n}^{(k)}, X_{n}^{(k)} \sqrt{n}\right)$.

Let $d_{n}^{-}$denote the in-degree of the node at the origin in this random directed graph under $\mathbb{P}^{0}$, namely the number of points whose ball contains the origin. Let $d_{n}^{+}$denote the out-degree of the origin, namely the number of points which fall in the ball of the point at the origin. From the mass transport principle [6], or by straightforward elementary arguments based on an ergodic theorem for spatial averages, we have

$$
\mathbb{E}_{n}^{0}\left[d_{n}^{+}\right]=\mathbb{E}_{n}^{0}\left[d_{n}^{-}\right] .
$$

Now, we have

$$
\begin{aligned}
\mathbb{E}_{n}^{0}\left[d_{n}^{+}\right] & \stackrel{(a)}{=} e^{n \rho_{n}} \mathbb{E}\left[\frac{(\pi n)^{\frac{n}{2}}}{\Gamma\left(\frac{n}{2}+1\right)} \bar{X}_{n}^{n}\right] \\
& =e^{n \rho_{n}} \mathbb{E}\left[e^{\frac{n}{2} \log (2 \pi e)+o(n)} e^{n \log \bar{X}_{n}}\right] \\
& =e^{n\left(\rho+\frac{1}{2} \log (2 \pi e)\right)+o(n)} \mathbb{E}\left[e^{n \log \bar{X}_{n}}\right] .
\end{aligned}
$$

Here step (a) follows from Slivnyak's theorem. Then we have the following result. 


\section{Lemma 7.}

$$
\frac{1}{n} \log \mathbb{E}_{n}^{0}\left[d_{n}^{-}\right] \rightarrow \rho+\frac{1}{2} \log (2 \pi e)+\sup _{R \geq R^{*}}(\log R-I(R)), \quad \text { as } n \rightarrow \infty .
$$

Proof. From what precedes,

$$
\mathbb{E}_{n}^{0}\left[d_{n}^{-}\right]=e^{n\left(\rho+\frac{n}{2} \log (2 \pi e)\right)+o(n)} \mathbb{E}\left[e^{n \log \bar{X}_{n}}\right] .
$$

It follows from Assumption (11) and from Varadhan's lemma [2] that

$$
\lim _{n \rightarrow \infty} \frac{1}{n} \mathbb{E}\left[e^{n \log \bar{X}_{n}}\right]=\sup _{R \geq R^{*}}(\log R-I(R)),
$$

where we have used the observation that $\log R-I(R) \leq \log R^{*}$ for $0 \leq R \leq R^{*}$. This completes the proof.

Now, from the independent thinning theorem [3, Exercise 11.3.1], the distribution of $d_{n}^{-}$is Poisson. Thus

$$
\mathbb{P}\left(\underline{0} \in \mathcal{C}_{n}\right)=1-\exp \left(-\mathbb{E}_{n}^{0}\left[d_{n}^{-}\right]\right) \leq \mathbb{E}_{n}^{0}\left[d_{n}^{-}\right] .
$$

For $\rho<\tau_{v}$, we see from eqn. (16) that

$$
\mathbb{E}\left[d_{n}^{-}\right] \rightarrow 0 \text { as } n \rightarrow \infty
$$

which implies that

$$
\mathbb{P}\left(\underline{0} \in \mathcal{C}_{n}\right) \rightarrow 0 \text { as } n \rightarrow \infty .
$$

In addition, for all $\alpha<1$ we have

$$
1-\alpha x \geq \exp (-x) \geq 1-x \text { for all sufficiently small } x>0 .
$$

Thus, from eqn. (17) we get that, for all $\alpha<1$ and $n$ sufficiently large

$$
\frac{1}{n} \log \mathbb{E}_{n}^{0}\left[d_{n}^{-}\right] \geq \frac{1}{n} \log \mathbb{P}\left(\underline{0} \in \mathcal{C}_{n}\right) \geq \frac{1}{n} \log \mathbb{E}_{n}^{0}\left[d_{n}^{-}\right]+\frac{1}{n} \log \alpha .
$$

Thus we have

$$
\lim _{n \rightarrow \infty} \frac{1}{n} \log P\left(\underline{0} \in \mathcal{C}_{n}\right)=\rho+\frac{1}{2} \log (2 \pi e)+\sup _{R \geq R^{*}}(\log R-I(R)),
$$

which concludes the proof of eqn. (4).

Suppose now that $\rho>\tau_{v}$ Since

$$
\mathbb{P}\left(\underline{0} \notin \mathcal{C}_{n}\right)=\exp \left(-\mathbb{E}_{n}^{0}\left[d_{n}^{-}\right]\right),
$$

we then immediately get eqn. (5). 


\subsection{Volume Fraction Threshold for Deterministic Radii}

The proof above also works for the case of deterministic radii (equal to $R_{n}^{*} \sqrt{n}$ in dimension $n$ with $R_{n}^{*} \rightarrow R^{*}$ as $\left.n \rightarrow \infty\right)$. The only change needed is to replace $\mathbb{E}\left[e^{n \log \bar{X}_{n}}\right]$ by $e^{n \log R_{n}^{*}}$. Then eqn. (16) is replaced by

$$
\frac{1}{n} \log \mathbb{E}_{n}^{0}\left[d_{n}^{-}\right] \rightarrow \rho+\frac{1}{2} \log (2 \pi e)+\log R^{*},
$$

and we learn that if

$$
\rho<-\frac{1}{2} \log (2 \pi e)-\log R^{*}
$$

then

$$
\lim _{n \rightarrow \infty} \frac{1}{n} \log \mathbb{P}\left(\underline{0} \in \mathcal{C}_{n}\right)=\rho+\frac{1}{2} \log (2 \pi e)+\log R^{*},
$$

which replaces eqn. (18), while if

$$
\rho>-\frac{1}{2} \log (2 \pi e)-\log R^{*}
$$

then

$$
\lim _{n \rightarrow \infty} \frac{1}{n} \log \left(-\log \mathbb{P}\left(\underline{0} \notin \mathcal{C}_{n}\right)\right)=\rho+\frac{1}{2} \log (2 \pi e)+\log R^{*},
$$

which replaces eqn. (5).

\subsection{Proof of Theorem 2}

In each dimension $n$, consider the stationary version of the process. Given $s>0$, let $N_{n}(\underline{0}, s)$ denote the number of points whose balls intersect the ball of radius $s \sqrt{n}$ centered at the origin $\underline{0}$ in $\mathbb{R}^{n}$. Then

$$
\begin{aligned}
\mathbb{E}\left[N_{n}(\underline{0}, s)\right] & \stackrel{(a)}{=} e^{n \rho_{n}} \mathbb{E}\left[\frac{(\pi n)^{\frac{n}{2}}}{\Gamma\left(\frac{n}{2}+1\right)}\left(\bar{X}_{n}+s\right)^{n}\right] \\
& =e^{n \rho_{n}} \mathbb{E}\left[e^{\frac{n}{2} \log (2 \pi e)+o(n)} e^{n \log \left(\bar{X}_{n}+s\right)}\right] \\
& =e^{n\left(\rho+\frac{1}{2} \log (2 \pi e)\right)+o(n)} \mathbb{E}\left[e^{n \log \left(\bar{X}_{n}+s\right)}\right] .
\end{aligned}
$$

Step (a) again follows from Slivnyak's theorem and the mass transport principle applied to the directed graph with an edge from $T_{n}^{(k)}$ to $T_{n}^{(l)}, l \neq k$ if $T_{n}^{(l)} \in$ $B\left(T_{n}^{(k)},\left(X_{n}^{(k)}+s\right) \sqrt{n}\right)$. 
Consider now the Palm version of the point process. Recall that, from Slivnyak's theorem, ignoring the point at $\underline{0}$ and its mark leaves behind a stationary version of the marked point process, which is called the reduced process. We therefore have,

$$
\mathbb{E}_{n}^{0}\left[D_{n}\right]=\mathbb{E}\left[\mathbb{E}\left[N_{n}(\underline{0}, S) \mid S\right]\right]
$$

where $S \stackrel{d}{=} \bar{X}_{n}$ and is independent of the reduced process.

Thus

$$
\mathbb{E}_{n}^{0}\left[D_{n}\right]=e^{n\left(\rho+\frac{1}{2} \log (2 \pi e)\right)+o(n)} \mathbb{E}\left[e^{n \log \left(\bar{X}_{n}+\bar{X}_{n}^{\prime}\right)}\right],
$$

with $\bar{X}_{n}$ and $\bar{X}_{n}^{\prime}$ being i.i.d. We now use the fact that $\bar{X}_{n}+\bar{X}_{n}^{\prime}$ satisfies an LDP with good convex rate function $2 I\left(\frac{u}{2}\right)$ to derive (9) from Varadhan's lemma. For this, we have to check that if $\bar{X}_{n}^{\prime}$ is a variable with the same law as $\bar{X}_{n}$ and such that $\bar{X}_{n}^{\prime}$ and $\bar{X}_{n}$ are independent, then

$$
\limsup _{n \rightarrow \infty} \mathbb{E}\left[\left(\bar{X}_{n}+\bar{X}_{n}^{\prime}\right)^{\gamma n}\right]^{\frac{1}{n}}<\infty \quad \text { for some } \gamma>1
$$

But this can be obtained from the following convexity argument

$$
\mathbb{E}\left[\left(\bar{X}_{n}+\bar{X}_{n}^{\prime}\right)^{\gamma n}\right] \leq 2^{\gamma n} \mathbb{E}\left[\left(\bar{X}_{n}\right)^{\gamma n}\right]
$$

which implies that

$$
\mathbb{E}\left[\left(\bar{X}_{n}+\bar{X}_{n}^{\prime}\right)^{\gamma n}\right]^{\frac{1}{n}} \leq 2^{\gamma} \mathbb{E}\left[\left(\bar{X}_{n}\right)^{\gamma n}\right]^{\frac{1}{n}}
$$

Hence eqn. (20) follows from eqn. (1).

This completes the proof of the results on the degree threshold.

\subsection{Degree Threshold for Deterministic Radii}

The proof given above also works for the case of deterministic radii. The changes needed are analogous to those that were needed in the case of the volume fraction threshold.

\subsection{Proof of Theorem 4}

We need to prove two things: (1) if $\rho<\tau_{p}$ then $\lim _{n \rightarrow \infty} \theta_{n}=0$ and (2) if $\rho>\tau_{p}$, then $\liminf \operatorname{int\infty }_{n \rightarrow \infty} \theta_{n}>0$. 
To prove (1) we follow the lines of the proof in Section 4.3. Rather than considering $\mathbb{E}_{n}^{0}\left[D_{n}\right]$, we consider $\mathbb{P}_{n}^{0}\left(D_{n}>0\right)$. As in Section 4.3 , we write

$$
\mathbb{P}_{n}^{0}\left(D_{n}>0\right)=\mathbb{E}_{n}^{0}\left[\mathbb{P}_{n}^{0}\left(D_{n}>0 \mid S\right)\right]
$$

where $S \sqrt{n}$ now refers to the radius of the ball of the point at the origin. Now, if

$$
\rho<-\frac{1}{2} \log (2 \pi e)+\inf _{R \geq R^{*}}\left(I(R)-\log \left(R+R^{*}\right)\right)
$$

then, because $R_{p}$, as defined in Section 3.4 is finite, for sufficiently small $\epsilon>0$ we also have

$$
\rho<-\frac{1}{2} \log (2 \pi e)+\inf _{R \geq R^{*}}\left(I(R)-\log \left(R+R^{*}+\epsilon\right)\right) .
$$

On the event $\left\{S \leq R^{*}+\epsilon^{\prime}\right\}$, with $\epsilon>\epsilon^{\prime}>0$, we have

$$
\begin{aligned}
& \lim _{n} \frac{1}{n} \log \left(\mathbb{E}_{n}^{0}\left[D_{n} \mid S\right]\right) \\
& =\rho+\frac{1}{2} \log (2 \pi e)+\sup _{R \geq R^{*}+S}(\log R-I(R-S)) \\
& =\rho+\frac{1}{2} \log (2 \pi e)+\sup _{\tilde{R} \geq R^{*}}(\log (\tilde{R}+S)-I(\tilde{R})) \\
& \leq \inf _{R \geq R^{*}}\left(I(R)-\log \left(R+R^{*}+\epsilon\right)\right)+\sup _{\tilde{R} \geq R^{*}}(\log (\tilde{R}+S)-I(\tilde{R})) \\
& =\sup _{\tilde{R} \geq R^{*}}(\log (\tilde{R}+S)-I(\tilde{R}))-\sup _{\tilde{R} \geq R^{*}}\left(\log \left(\tilde{R}+R^{*}+\epsilon\right)-I(\tilde{R})\right) \\
& <0
\end{aligned}
$$

We also have

$$
\begin{aligned}
\mathbb{P}_{n}^{0}\left(D_{n}>0\right)= & \mathbb{P}_{n}^{0}\left(D_{n}>0, S>R^{*}+\epsilon^{\prime}\right) \\
& +\mathbb{E}_{n}^{0}\left[\mathbb{P}_{n}^{0}\left(D_{n}>0 \mid S\right) 1\left(S \leq R^{*}+\epsilon^{\prime}\right)\right] \\
\leq & \mathbb{P}\left(S>R^{*}+\epsilon^{\prime}\right)+\mathbb{E}_{n}^{0}\left[\mathbb{E}_{n}^{0}\left[D_{n} \mid S\right] 1\left(S \leq R^{*}+\epsilon^{\prime}\right)\right]
\end{aligned}
$$

In the last expression, the first term has probability asymptotically approaching 0 as $n \rightarrow \infty$. From eqn. (21), for all $s$ in the integration interval, the integrand in the second term tends pointwise to 0 as $n \rightarrow \infty$. From this and dominated convergence, we conclude that $\mathbb{P}_{n}^{0}\left(D_{n}>0\right) \rightarrow 0$ as $n \rightarrow \infty$, which proves (1). 
We now prove (2), assuming that

$$
\rho>-\frac{1}{2} \log (2 \pi e)+\inf _{R \geq R^{*}}\left(I(R)-\log \left(R+R^{*}+\epsilon\right)\right) .
$$

Let $R_{p}$, as defined in Section 3.4 achieve the minimum in the definition of $\tau_{p}$ in eqn. (13). We need to distinguish between the two cases $R_{p}=R^{*}$ and $R_{p}>R^{*}$.

Consider first the case $R_{p}=R^{*}$. Then

$$
\rho=-\frac{1}{2} \log (2 \pi e)-\log \left(2 R^{*}\right)+\delta,
$$

for some $\delta>0$. This means that we can choose $\gamma>0$ such that

$$
\rho>-\frac{1}{2} \log (2 \pi e)-\log \left(2\left(R^{*}-\gamma\right)\right)+\frac{\delta}{2} .
$$

For each dimension $n$ we consider the thinned version of the reduced process, where we only retain the points whose balls have radius at least $\left(R^{*}-\gamma\right) \sqrt{n}$. We also consider only the event that the ball of the point at the origin has radius at least $\left(R^{*}-\gamma\right) \sqrt{n}$.

Let $\tilde{\theta}_{n}$ denote the probability of percolation from the origin via its ball and through the balls of the thinned reduced point process, on the event that the ball of the origin has radius at least $\left(R^{*}-\gamma\right) \sqrt{n}$. Since $\theta_{n} \geq \tilde{\theta}_{n}$, if we can show that $\liminf _{n \rightarrow \infty} \tilde{\theta}_{n}>0$, then we will be done.

Let us now show that

(a) the probability that the ball of $\underline{0}$ has radius at least $\left(R^{*}-\gamma\right) \sqrt{n}$ tends to 1 as $n$ tends to infinity;

(b) the probability that the number $J_{n}$ of balls of the thinned point process intersecting the ball of $\underline{0}$ (with radius at least $\left(R^{*}-\gamma\right) \sqrt{n}$ ) is positive tends to 1 as $n$ tends to infinity;

(c) the Boolean model with deterministic radii $\left(R^{*}-\gamma\right) \sqrt{n}$ for the points of the thinned reduced process percolates, i.e. the associated percolation probability has a positive liminf.

Property (a) is immediate. For proving (b), we show that $\mathbb{E}\left[J_{n}\right]$ tends to infinity with $n$, which will complete the proof since $J_{n}$ is Poisson. The probability that 
the $\bar{X}_{n}$ exceeds $\left(R^{*}-\gamma\right) \sqrt{n}$ is asymptotically 1 . Hence, by arguments similar to those used earlier,

$$
\lim _{n} \frac{1}{n} \mathbb{E}\left[J_{n}\right]=\rho+\frac{1}{2} \log (2 \pi e)+\ln \left(2\left(R^{*}-\gamma\right)\right)>\frac{\delta}{2},
$$

which completes the proof of (b). For proving (c), we use the results in [8]. The percolation threshold of the Boolean models with deterministic radii $\left(R^{*}-\gamma\right) \sqrt{n}$ is $-\frac{1}{2} \log (2 \pi e)-\log \left(2\left(R^{*}-\gamma\right)\right)$. Since the normalized logarithmic intensity of the thinned reduced process is still asymptotically $\rho$, and since this exceeds $-\frac{1}{2} \log (2 \pi e)-\log \left(2\left(R^{*}-\gamma\right)\right)$, the proof of (c) follows from [8].

The proof of the desired result, in the case $R_{p}=R^{*}$, now follows immediately from (a), (b), and (c).

We next turn to the case $R_{p}>R^{*}$. Note that in this case we must have $I\left(R_{p}\right)<\infty$. Since

$$
\rho=-\frac{1}{2} \log (2 \pi e)+I\left(R_{p}\right)-\log \left(R_{p}+R^{*}\right)+\delta,
$$

for some $\delta>0$, we can choose $\gamma>0$ such that $R^{*}<R_{p}-\gamma<R_{p}$ (which implies that $\left.I\left(R_{p}-\gamma\right)<\infty\right)$, and such that

$$
\rho>-\frac{1}{2} \log (2 \pi e)+I\left(R_{p}-\gamma\right)-\log \left(R_{p}+R^{*}-2 \gamma\right)+\frac{\delta}{2} .
$$

For each dimension $n$ we consider the thinned version of the reduced process, where we only retain the points whose balls have radius at least $\left(R_{p}-\gamma\right) \sqrt{n}$. We also consider only the event that the ball of the point at the origin has radius at least $\left(R^{*}-\gamma\right) \sqrt{n}$.

Let $\tilde{\theta}_{n}$ denote the probability of percolation from the origin via its ball and through the balls of the thinned reduced point process, on the event that the ball of the origin has radius at least $\left(R^{*}-\gamma\right) \sqrt{n}$. Since $\theta_{n} \geq \tilde{\theta}_{n}$, if we can show that $\liminf _{n \rightarrow \infty} \tilde{\theta}_{n}>0$, then we will be done.

Let us now show that

(a) the probability that the ball of $\underline{0}$ has radius at least $\left(R^{*}-\gamma\right) \sqrt{n}$ tends to 1 as $n$ tends to infinity;

(b) the probability that the number $J_{n}$ of balls of the thinned point process intersecting the ball of $\underline{0}$ (with radius at least $\left(R^{*}-\gamma\right) \sqrt{n}$ ) is positive tends to 1 as $n$ tends to infinity; 
(c) the Boolean model with deterministic radii $\left(R_{p}-\gamma\right) \sqrt{n}$ for the points of the thinned reduced process percolates, i.e. the associated percolation probability has a positive liminf.

Property (a) is immediate. For proving (b), we show that $\mathbb{E}\left[J_{n}\right]$ tends to infinity with $n$, which will complete the proof since $J_{n}$ is Poisson. The probability that the $\bar{X}_{n}$ exceeds $\left(R_{p}-\epsilon\right) \sqrt{n}$ scales like $e^{-n I\left(R_{p}-\gamma\right)+o(n)}$. Hence, by arguments similar to those used earlier,

$$
\left.\lim _{n} \frac{1}{n} \mathbb{E}\left[J_{n}\right]=\rho+\frac{1}{2} \log (2 \pi e)-I\left(R_{p}-\gamma\right)+\ln \left(R_{p}+R^{*}-2 \gamma\right)\right)>\frac{\delta}{2},
$$

which completes the proof of (b). For proving (c), we use the results in [8]. The percolation threshold of the Boolean models with deterministic radii $\left(R_{p}-\gamma\right) \sqrt{n}$ is $-\frac{1}{2} \log (2 \pi e)-\log \left(2\left(R_{p}-\gamma\right)\right)$. Since the normalized logarithmic intensity of the thinned reduced process is asymptotically $\rho-I\left(R_{p}-\gamma\right)$, and since this exceeds $-\frac{1}{2} \log (2 \pi e)-\log \left(R_{p}+R^{*}-2 \gamma\right)$ ) (because $R_{p}>R^{*}$ ), the proof of (c) follows from [8].

The proof of the desired result, in the case $R_{p}>R^{*}$, now follows immediately from (a), (b), and (c). This also completes the overall proof.

\subsection{Percolation Threshold for Deterministic Radii}

The proof of the percolation threshold in the case of deterministic radii (i.e. when the radii in dimension $n$ equal a constant $R_{n}^{*} \sqrt{n}$, with $R_{n}^{*} \rightarrow R^{*}$ as $n \rightarrow \infty$ ) can be completed in a much simpler way than the general proof. Since $\tau_{p}=\tau_{d}$ in the case of deterministic radii, the absence of percolation when $\rho<\tau_{p}$ is an immediate consequence of the result proved earlier that $\mathbb{P}\left(D_{n}>0\right) \rightarrow 0$ as $n \rightarrow \infty$ when $\rho<\tau_{d}$. For the case $\rho>\tau_{p}$ the proof can be done in a way exactly as the general case where $R_{p}=R^{*}$ was handled above.

\subsection{Proof of Theorem 5}

We first prove that $\tau_{d} \leq \tau_{p}$. By the convexity of the rate function $I(\cdot)$, and because $I\left(R^{*}\right)=0$, we have, for all $R \geq R^{*}$,

$$
2 I\left(\frac{R+R^{*}}{2}\right) \leq I(R)
$$


Hence, for all $R \geq R^{*}$,

$$
2 I\left(\frac{R+R^{*}}{2}\right)-\log \left(2\left(\frac{R+R^{*}}{2}\right)\right) \leq I(R)-\log \left(R+R^{*}\right) .
$$

This establishes the result.

The fact that $\tau_{p} \leq \tau_{v}$ immediately follows from

$$
\inf _{R \geq R^{*}}\left(I(R)-\log \left(R+R^{*}\right)\right) \leq \inf _{R \geq R^{*}}(I(R)-\log (R)) .
$$

\subsection{Proof of Theorem 6}

The fact that $R^{*} \leq R_{d}$ is immediate from the definition of $R_{d}$.

We first prove that $R_{d} \leq R_{p}$. Recall that $R_{d}$ is uniquely defined by

$$
\frac{1}{2 R_{d}} \in\left[I_{-}^{\prime}\left(R_{d}\right), I_{+}^{\prime}\left(R_{d}\right)\right]
$$

and $R_{p}$ is uniquely defined by

$$
\frac{1}{R_{p}+R^{*}} \in\left[I_{-}^{\prime}\left(R_{p}\right), I_{+}^{\prime}\left(R_{p}\right)\right]
$$

Now

$$
R_{d}>R_{p} \Longrightarrow 2 R_{d}>R_{p}+R^{*} \Longrightarrow \frac{1}{2 R_{d}}<\frac{1}{R_{p}+R^{*}} \Longrightarrow R_{d}<R_{p} .
$$

This contradiction implies that $R_{d} \leq R_{p}$.

We next prove that $R_{p} \leq R_{v}$. Here we also need to recall that $R_{v}$ is uniquely defined by

$$
\frac{1}{R_{v}} \in\left[I_{-}^{\prime}\left(R_{v}\right), I_{+}^{\prime}\left(R_{v}\right)\right]
$$

Now

$$
R_{p}>R_{v} \Longrightarrow R_{p}+R^{*}>R_{v} \Longrightarrow \frac{1}{R_{p}+R^{*}}<\frac{1}{R_{v}} \Longrightarrow R_{p}<R_{v} .
$$

This contradiction proves that $R_{p} \leq R_{v}$.

We next prove that $R_{v} \leq R_{p}+R^{*}$. For this, it suffices to observe the contradiction

$$
R_{v}>R_{p}+R^{*} \Longrightarrow \frac{1}{R_{v}}<\frac{1}{R_{p}+R^{*}} \Longrightarrow R_{v}<R_{p} \Longrightarrow R_{v}<R_{p}+R^{*}
$$


We finally prove that $R_{p}+R^{*} \leq 2 R_{d}$. For this we observe the contradiction $R_{p}+R^{*}>2 R_{d} \Longrightarrow \frac{1}{R_{p}+R^{*}}<\frac{1}{2 R_{d}} \Longrightarrow R_{p}<R_{d} \Longrightarrow R_{p}+R^{*}<2 R_{d}$.

This completes the proof.

\section{Concluding Remarks}

\subsection{Connections with Error Exponents}

In this subsection we make some remarks about the connections between the concerns of this paper and the problem of error exponents in channel coding over the additive white Gaussian noise channel [4, Section 7.4], as discussed in [1] in the Poltyrev regime.

For all $n \geq 1$ and $k \geq 1$, let $W_{n}^{(i, k)}, n \geq i \geq 1$, denote an i.i.d. sequence of Gaussian random variables, all centered and of variance $\sigma^{2}$. Let $W_{n}^{(k)}$ denote the $n$-dimensional vector with coordinates $W_{n}^{(i, k)}, n \geq i \geq 1$. Then $T_{n}^{(k)}+W_{n}^{(k)}$ belongs to the closed ball of center $T_{n}^{(k)}$ and radius $X_{n}^{(k)} \sqrt{n}$, with

$$
X_{n}^{(k)}:=\left(\frac{1}{n} \sum_{i=1}^{n}\left(W_{n}^{(i, k)}\right)^{2}\right)^{\frac{1}{2}} \stackrel{d}{=} \bar{X}_{n}
$$

where $\bar{X}_{n}$ denotes a random variable having the distribution of the normalized radius random variables in the preceding equation. One can check that $\left(\bar{X}_{n}, n \geq\right.$ 1) satisfy an LDP and all the assumptions listed above. For each $\sigma^{2}>0$, we call such a family of Boolean models (parametrized by $n \geq 1$, as usual) the case with Gaussian grains.

For Shannon's channel coding problem in the Poltyrev regime, as considered in [1], the focus is on the probability of error. As a result, one only wants to associate those points in Euclidean space that have a high probability of being of the type $T_{n}^{(k)}+W_{n}^{(k)}$ to the point $T_{n}^{(k)}$. Therefore one considers, instead of the Boolean model where a Gaussian grain is associated to each point, another Boolean model where this Gaussian grain is replaced by an associated typicality region, namely the set

$$
\left\{T_{n}^{(k)}+v: v \in \mathbb{R}^{n},\|v\|_{2} \leq \sigma \sqrt{n}+\alpha_{n}\right\}
$$


where $\|v\|_{2}$ denotes the usual Euclidean length of $v$ and where $0<\alpha_{n}=O(\sqrt{n})$ are chosen such that

$$
\begin{aligned}
& \frac{\alpha_{n}}{\sqrt{n}} \rightarrow 0 \text { as } n \rightarrow \infty ; \\
& \left.P\left(\left\|W_{n}^{(k)}\right\| \leq \sigma \sqrt{n}+\alpha_{n}\right) \rightarrow 1 \text { as } n \rightarrow \infty \text { (for each } 1 \leq k \leq n\right) ; \\
& \frac{1}{n} \log \operatorname{Vol}\left\{v \in \mathbb{R}^{n}:\|v\|_{2} \leq \sigma \sqrt{n}+\alpha_{n}\right\} \rightarrow \frac{1}{2} \log \left(2 \pi e \sigma^{2}\right) \text { as } n \rightarrow \infty .
\end{aligned}
$$

This now gives rise to a family of deterministic Boolean models which will be referred to as the truncated Gaussian grain models below. The Poltyrev capacity is the threshold for the asymptotic logarithmic intensity of such a family of Boolean models. This threshold is the asymptotic logarithmic intensity up to which it is possible to make such an association with asymptotically vanishing probability of error. It is also the threshold up to which there is a vanishingly small probability that a point in Euclidean space is covered by multiple truncated Gaussian grains, which directly corresponds to what is called the volume fraction threshold in the present paper.

It turns out that the volume fraction threshold is smaller for Gaussian grains than for truncated Gaussian grains, even though the normalized radii of the grains in the two models have the same asymptotic limit $R^{*}$. To see this, consider Gaussian grains with per-coordinate variance $\sigma^{2}$, as above. Then $\bar{X}_{n}^{2}$ is distributed as the average of $n$ i.i.d. squared Gaussian random variables of mean 0 and variance $\sigma^{2}$, so we have $\bar{X}_{n}^{2} \stackrel{\mathbb{P}}{\rightarrow} \sigma^{2}$ as $n \rightarrow \infty$, which implies $\bar{X}_{n} \stackrel{\mathbb{P}}{\rightarrow} \sigma$ as $n \rightarrow \infty$. This means $E\left[\bar{X}_{n}\right]$ (which is bounded above by $\left(E\left[\left(\bar{X}_{n}\right)^{2}\right]\right)^{\frac{1}{2}}=\sigma$ ) converges to $\sigma$ as $n \rightarrow \infty$. This means $R^{*}=\sigma$. The volume fraction threshold for deterministic grains with radius $R^{*} \sqrt{n}$ in dimension $n$ is then given by the R.H.S. of eqn. (6). The exponent of the growth rate in $n$ of the volume of each Gaussian grain is strictly bigger than this. That it is at least as big follows immediately from the convexity of the function $R \mapsto R^{n}$, defined for $R \geq 0$. To see the strict inequality, first note that the density of the radius of the Gaussian grain in dimension $n$ can be written as $g_{n}^{\sigma}(r), r \geq 0$, where $g_{n}^{\sigma}(r)=\frac{1}{\sigma} g_{n}^{1}\left(\frac{r}{\sigma}\right)$, with

$$
g_{1}^{\sigma}(r):=\frac{n r^{n-1} e^{-\frac{r^{2}}{2}}}{2^{\frac{n}{2}} \Gamma\left(\frac{n}{2}+1\right)}, r \geq 0,
$$

where $\Gamma(\cdot)$ denotes the standard Euler gamma function. To figure out the asymptotic growth rate of the expected volume of a grain, we need to compute

$$
\lim _{n \rightarrow \infty} \frac{1}{n} \log \int_{0}^{\infty} V_{n}(1) r^{n} g_{n}^{\sigma}(r) d r
$$


where $V_{n}(1):=\frac{\pi^{\frac{n}{2}}}{\Gamma\left(\frac{n}{2}+1\right)}$ denotes the volume of the ball of unit radius in $\mathbb{R}^{n}$.

It is convenient to reparametrize $r$ as $v \sigma \sqrt{n}$, giving

$$
g_{n}^{\sigma}(v \sigma \sqrt{n})=e^{-n\left(\frac{v^{2}}{2}-\frac{1}{2}-\log (v)+o(1)\right)} .
$$

Thus

$$
\begin{aligned}
& \lim _{n \rightarrow \infty} \frac{1}{n} \log \int_{0}^{\infty} \frac{\pi^{\frac{n}{2}}}{\Gamma\left(\frac{n}{2}+1\right)} r^{n} g_{n}^{\sigma}(r) d r \\
= & \lim _{n \rightarrow \infty} \frac{1}{n} \log \left((2 \pi e \sigma)^{\frac{n}{2}} \int_{0}^{\infty} e^{n(\log v+o(1))} e^{-n\left(\frac{v^{2}}{2}-\frac{1}{2}-\log (v)+o(1)\right)} d v\right) \\
\stackrel{(a)}{=} & \frac{1}{2} \log \left(2 \pi e \sigma^{2}\right)+\frac{1}{2}(\log 4-1) \\
> & \frac{1}{2} \log \left(2 \pi e \sigma^{2}\right),
\end{aligned}
$$

where step (a) comes from Laplace's principle that the asymptotics is controlled by the exponential term in the integrand with the largest exponent. Laplace's principle as just applied is only a heuristic, of course, but this calculation makes the point that the volume fraction threshold for Gaussian grains is strictly smaller than the volume fraction threshold for the truncated Gaussian grains (i.e. the Poltyrev threshold).

\subsection{Thresholds in the Gaussian Grain Case}

It is interesting to consider the case of Gaussian grains in detail as an illustration of the general results in this paper, and we turn to this next, giving, in the process, a rigorous derivation of the volume fraction threshold for this case as discussed in the preceding subsection.

We first need to determine the large deviations rate function for the sequence $\left(\bar{X}_{n}, n \geq 1\right)$, with each $\bar{X}_{n}$ defined as in eqn. (22). Here we think of $\sigma^{2}>0$ as being fixed. It is easy to do this by first observing that $\left(\bar{X}_{n}^{2}, n \geq 1\right)$ satisfies the large deviations principle with rate function $J(\cdot)$ given by

$$
J(z)= \begin{cases}\frac{z}{2 \sigma^{2}}-\frac{1}{2}-\frac{1}{2} \log \frac{z}{\sigma^{2}} & \text { if } z>0 \\ \infty & \text { otherwise }\end{cases}
$$


which follows from the fact that if $Z_{n}$ is a Gaussian random variable with mean zero and variance $\sigma^{2}$ then

$$
\log E\left[e^{\theta Z_{n}^{2}}\right]= \begin{cases}-\frac{1}{2} \log \left(1-2 \theta \sigma^{2}\right) & \text { if } \theta<\frac{1}{2 \sigma^{2}} \\ \infty & \text { otherwise }\end{cases}
$$

The contraction principle [2, Thm. 4.2.1] then gives the rate function of the sequence $\left(\bar{X}_{n}, n \geq 1\right)$ as being $I(\cdot)$, where

$$
I(R)= \begin{cases}\frac{R^{2}}{2 \sigma^{2}}-\frac{1}{2}-\frac{1}{2} \log \frac{R^{2}}{\sigma^{2}} & \text { if } R>0 \\ \infty & \text { otherwise }\end{cases}
$$

Another way to see this is to note that the convex conjugate dual of this function is the function

$$
\Lambda(\theta)=\frac{\theta \sigma}{2}\left(\frac{\theta \sigma+\sqrt{\theta^{2} \sigma^{2}+4}}{2}\right)+\log \left(\frac{\theta \sigma+\sqrt{\theta^{2} \sigma^{2}+4}}{2}\right),
$$

and to check that

$$
\begin{aligned}
\Lambda(\theta) & =\lim _{n \rightarrow \infty} \frac{1}{n} \log E\left[e^{n \theta \bar{X}_{n}}\right] \\
& =\lim _{n \rightarrow \infty} \frac{1}{n} \log \int_{0}^{\infty} e^{\sqrt{n} \theta r} g_{n}^{\sigma}(r) d r \\
& =\lim _{n \rightarrow \infty} \frac{1}{n} \log \int_{0}^{\infty} e^{n\left(\theta v \sigma-\frac{v^{2}}{2}+\frac{1}{2}+\log v+o(1)\right)} d v,
\end{aligned}
$$

by the use of Laplace's principle in the last expression on the right hand side.

The volume fraction threshold for the case of Gaussian grains (with $\sigma^{2}>0$ being fixed) it then given by finding the solution $R_{v} \geq \sigma$ to the equality

$$
\frac{R}{\sigma^{2}}-\frac{1}{R}=\frac{1}{R}
$$

There is a unique solution to this equation, namely $R_{v}=\sigma \sqrt{2}$, and this turns out to satisfy $R_{v} \geq \sigma$, as it should. Here $\sigma$ is playing the role of $R^{*}$ in the general theory. Substituting back into the formula

$$
\tau_{v}=-\frac{1}{2} \log (2 \pi e)+I\left(R_{v}\right)-\log \left(R_{v}\right)
$$


for the volume fraction threshold gives

$$
\tau_{v}=-\frac{1}{2} \log \left(2 \pi e \sigma^{2}\right)-\frac{1}{2}(\log 4-1) .
$$

This is the announced rigorous derivation of the formula that was found above by the heuristic application of Laplace's principle.

The degree threshold is given by finding the solution $R_{d} \geq \sigma$ to the equality

$$
\frac{R}{\sigma^{2}}-\frac{1}{R}=\frac{1}{2 R}
$$

There is a unique solution to this equation, namely $R_{d}=\sigma \sqrt{\frac{3}{2}}$, and this turns out to satisfy $R_{d} \geq \sigma$, as it should. Substituting back into the formula

$$
\tau_{d}=-\frac{1}{2} \log (2 \pi e)+2 I\left(R_{d}\right)-\log \left(2 R_{d}\right)
$$

for the degree threshold gives

$$
\tau_{d}=-\frac{1}{2} \log \left(2 \pi e \sigma^{2}\right)-\frac{1}{2}\left(\log \frac{27}{2}-1\right) .
$$

The percolation threshold is given by finding the solution $R_{p} \geq \sigma$ to the equality

$$
\frac{R}{\sigma^{2}}-\frac{1}{R}=\frac{1}{R+\sigma} .
$$

There is a unique solution to this equation, namely $R_{p}=\sigma c$, where $c$ is the unique root of the equation

$$
c^{3}+c^{2}-2 c-1=0,
$$

which satisfies $c \geq 0$. In fact, this root satisfies $c>1$. 4 Substituting back into the formula

$$
\tau_{p}=-\frac{1}{2} \log (2 \pi e)+I\left(R_{p}\right)-\log \left(R_{p}+\sigma\right)
$$

for the percolation threshold gives

$$
\tau_{p}=-\frac{1}{2} \log \left(2 \pi e \sigma^{2}\right)-\frac{1}{2}\left(\log \left(c^{2}(1+c)^{2}\right)-c^{2}+1\right) .
$$

\footnotetext{
${ }^{4}$ That there is a unique such root and that it satisfies $c>1$ can be verified by noting that the expression on the left hand side of this equation equals -1 at $c=0$ and at $c=1$ and goes to $\infty$ as $c \rightarrow \infty$ and, further, the derivative in $c$ of the expression is $3 c^{2}+2 c-2$, which equals -2 at $c=0$ and is a convex function.
} 
Numerical evaluation of $c$ gives $1.2469796<c<1.2469797$. This approximation suffices to verify that

$$
\log \left(\frac{27}{2}\right)-1>\log \left(c^{2}(1+c)^{2}\right)-c^{2}+1>\log (4)-1
$$

which confirms that $\tau_{d} \leq \tau_{p} \leq \tau_{v}$ in the case of Gaussian grains, as required by Theorem 5. This approximation also suffices to verify that

$$
1<\sqrt{\frac{3}{2}}<c<\sqrt{2}<1+c<\sqrt{6},
$$

which confirms that

$$
R^{*} \leq R_{d} \leq R_{p} \leq R_{v} \leq R_{p}+R^{*} \leq 2 R_{d},
$$

in the case of Gaussian grains, as required by Theorem 6 ,

Returning to the Boolean model with truncated Gaussian grains discussed in the last subsection, since, for every $\sigma^{2}>0$, this family of Boolean models is a deterministic model with $R^{*}=\sigma$, the rate function for this model satisfies

$$
I(\sigma)=0, \text { and } I(R)=\infty \text { for all } R \neq \sigma .
$$

Thus, in this case the deterministic threshold equals the percolation threshold, and both are $\log 2$ below the volume fraction threshold. It is interesting to note, as observed in [9] and [1], that this threshold also has a meaning; it is the threshold for the asymptotic logarithmic intensity up to which the truncated Gaussian grain of any given point of the Poisson process is so small that with probability asymptotically equal to 1 it does not meet the grain of any other point of the Poisson process. This feature, which relates to a study of pairwise conflict in decoding between two codewords, is central to Gallager's analysis of error exponents in the power constrained channels that are of interest to engineers; for more details see Section 7.4 of [4] and in particular the study there of what is called Gallager's $E_{0}$ function.

\section{Acknowledgements}

The research of the first author was supported by the ARO MURI grant W911NF08-1-0233, Tools for the Analysis and Design of Complex Multi-Scale Networks, the NSF grants CNS-0910702 and ECCS-1343398, and the NSF Science \& Technology Center grant CCF-0939370, Science of Information. This work of the second author was supported by an award from the Simons Foundation (\# 197982 to The University of Texas at Austin). 


\section{References}

[1] V. Anantharam and F. Baccelli, Capacity and Error Exponents of Stationary Point Processes under Random Additive Displacements, to appear in $\mathrm{Ad}$ vances in Applied Probability.

[2] A. Dembo and O. Zeitouni, Large Deviation Techniques and Applications, Jones and Bartlett, Boston, 1993.

[3] D. J. Daley and D. Vere-Jones, An Introduction to the Theory of Point Processes, Vol. 2, Second Edition, Springer, 2008.

[4] R. G. Gallager, Information Theory and Reliable Communications, John Wiley \& Sons, 1968.

[5] J.-B. Gouéré and R. Marchand, Continuum percolation in high dimensions, http://arxiv.org/abs/1108.6133, 2011.

[6] G. Last and H. Thorisson, Invariant transports of stationary random measures and mass-stationarity, The Annals of Probability, Pages 790-813, Vol. 37, Number 2, 2009.

[7] R. Meester and R. Roy, Continuum Percolation. Cambridge University Press, 1996.

[8] M. D. Penrose, Continuum Percolation and Euclidean Minimal Spanning Trees in High Dimensions. The Annals of Applied Probability, Vol. 6, No. 2 , pp. $528-544,1996$.

[9] G. Poltyrev, "On Coding Without Restrictions for the AWGN Channel". IEEE Trans. on Inform. Theory, Vol. 40, No. 2, pp. 409-417, Mar. 1994.

[10] R. T. Rockafellar, Convex Analysis, Princeton University Press, 1972.

[11] R. Schneider and W. Weil, Stochastic and Integral Geometry, Springer Verlag, 2008.

[12] S.R.S. Varadhan, Large Deviations and Applications, SIAM, 1984. 